\title{
Teaching Learning based Optimization Algorithm to Solve Assembly Line Balancing Problem
}

\author{
Shubham V. Kothavade ${ }^{\dagger}$ and S.P. Deshpande ${ }^{\dagger}$ \\ †Mechanical Engineering, GES's R.H.Sapat COE, Nashik,Maharastra, India \\ Accepted 01 Sept 2016, Available online 02 Sept 2016, Vol.6, No.5 (Oct 2016)
}

\begin{abstract}
Modern assembly lines are used in automobile industries in order to produce high quality and very complex products. These industries involve large number of input parameters which may affect cost and quality of final product. Selection of optimum parameters and process is very important objective in the present work. The mathematical models of assembly line of Scorpio (W105) considered for the optimization of respective assembly line. A recently developed advanced optimization algorithm named as teaching-learning-based optimization (TLBO) is used for the parameters optimization of the assembly line, which is inspired by teaching-learning process and works on the effect of influence of teacher on output of learners in class. This technique is used to minimize the computational efforts and considerable improvements in results are obtained in the problem near to optimum results. We also provided a comprehensive comparative study along with statistical analyses in order to present effectiveness of TLBO algorithm on solving scheduling problems. Experimental results show that the TLBO algorithm has a considerable potential when compared to the best-known heuristic algorithms for scheduling problem.
\end{abstract}

Keywords: Assembly line, Balancing, Teaching learning based Optimization, algorithm.

\section{Introduction}

An assembly line is a sequential of workstations that are connected by material handling system and is commonly used in producing high quality products (Hindriyanto Dwi Purnomo, Hui-Ming Wee 2014). The desired performance of an assembly line can be controlled by assigning tasks to workstations in such a way the assembly objective is fulfilled, the demand is met and the constraints imposed on the line are satisfied. Assembly lines are used for effective mass production with homogenous standard (Ana S. Simaria, Pedro M. Vilarinho 2009). The configuration of assembly lines is important due to the higher investment cost and its advantages to achieve the competitiveness of product cost. Based on the task operation direction, assembly line can be classified into one-side assembly lines (OALBP) or two-sided assembly lines (TALBP). OALBP is the most widely studied of line balancing problem (Ana S. Simaria, Pedro M. Vilarinho 2009).

The first study on two-sided assembly lines was written by Bartholdi (1993), who conducted an interactive program with balancing algorithm using the first fit heuristic. Kim et al. (2000) and Kim et al. (2009) used genetic algorithm to solve two assembly

*Corresponding author Shubham V. Kothavade is a Student and S.P. Deshpande B is working as Assistant Professor line balancing problems. Lee et al. (2001) proposed a group assignment procedure focusing on the maximization of work relatedness and work slackness. Simaria and Vilarinho (2009) implemented ant colony optimization for mixed two-sided assembly line balancing. Özcan (2010) applied simulated annealing for stochastic two-sided assembly line balancing (Parames Chutima Wanwisa Naruemitwong (2014).

Teaching-learning-based optimization algorithm (TLBO) is a teaching-learning process inspired algorithm recently proposed by Rao et al. $(2011,2012)$ and Rao and Patel (2012) based on the effect of influence of a teacher on the output of learners in a class [3]. The algorithm mimics teaching-learning ability of teacher and learners in a class room. Teacher and learners are the two vital components of the algorithm and describes two basic modes of the learning, through teacher (known as teacher phase) and interacting with the other learners (known as learner phase). The output in TLBO algorithm is considered in terms of results or grades of the learners which depend on the quality of teacher. A high quality teacher is usually considered as a highly learned person who trains learners so that they can have better results in terms of their marks or grades. Moreover, learners also learn from the interaction among themselves which also helps in improving their results. TLBO is population based method. In this algorithm a group of learners are considered as population and 
different subjects offered to the learners are considered as different design parameters and a learner's result is analogous to the 'fitness' value of the optimization problem. The best solution in the entire population is considered as the teacher. The design parameters are actually the parameters involved in the objective function of the given optimization problem and the best solution is the best value of the objective function. The working of TLBO is divided into two parts, 'Teacher phase' and 'Learner phase'.

\section{Teaching-learning-based optimization}

Teaching-Learning-Based Optimization (TLBO), is proposed in this paper for the 'Optimization of Assembly Line And Line Balancing'. This method works on the effect of influence of a teacher on learners. Like other nature-inspired algorithms, TLBO is also a population-based method and uses a population of solutions to proceed to the global solution. The population is considered as a group of learners or a class of learners. The process of TLBO is divided into two parts: the first part consists of the 'Teacher Phase' and the second part consists of the 'Learner Phase'.

'Teacher Phase' means learning from the teacher and 'Learner Phase' means learning by the interaction between learners. The basic philosophy of the TLBO Algorithm is explained in detail as follows:

Teacher phase:- A good teacher is one who brings his or her learners up to his or her level in terms of knowledge. But in practice this is not possible and a teacher can only move the mean of a class up to some extent depending on the capability of the class. This follows a random process depending on many factors. It can be explained as follows

Let $M(i)$ be the mean and $T(i)$ be the teacher at any iteration(i). T(i) will try to move mean M(i) towards its own level, so now the new mean will be T(i) designated as Mnew. The solution is updated according to the difference between the existing and the new mean given by

\section{Difference Mean(i) = r(i) [Mnew TF $\times$ M(i)]}

where

ri is a random number in the range 0 to 1 and

$\mathrm{TF}$ is a teaching factor that decides the value of mean to be changed and can be either 1 or 2 (which is a heuristic step and decided randomly)

This difference modifies the existing solution as given by the following equation

Xnew,(i) = Xold,(i) + Difference Mean(i).

Learner phase:-Learners increase their knowledge by two different means: one through input from the teacher and the other through interaction between themselves. A learner interacts randomly with other learners with the help of group discussions, presentations, formal communications, etc. A learner learns something new if the other learner has more knowledge than him or her. Learner modification is expressed as

For $\mathrm{i}=1:$ Pn

Randomly select two learners $\mathrm{Xi}$ and $\mathrm{Xj}$, where $\mathrm{i} \neq \mathrm{j}$

If $f(X i)<f(X j)$

$\mathrm{Xnew}(\mathrm{i})=\mathrm{Xold}(\mathrm{i})+\operatorname{ri}(\mathrm{Xi}-\mathrm{Xj})$

Else

Xnew $(\mathrm{i})=\mathrm{Xold}(\mathrm{i})+\mathrm{ri}(\mathrm{Xj}-\mathrm{Xi})$

Accept Xnew if it gives a better function value.

\section{Implementation of TLBO for optimization}

The step-wise procedure for the implementation of TLBO is explained as follows

Step 1: Define the optimization problem and initialize the

Optimization parameters.

Initialize the population size (Pn), number of generations $(\mathrm{Gn})$,

Number of design variables (Dn), and limits of design variables(UL, LL).

Define the optimization problem as: Minimize $\mathrm{f}(\mathrm{X})$.

Subject to $\mathrm{Xi} \in \mathrm{xi}=1,2 \ldots \mathrm{Dn}$

Where $\mathrm{f}(\mathrm{X})$ is the objective function, $\mathrm{X}$ is a vector for design variables such that $\mathrm{LL} \leq \mathrm{x}(\mathrm{i}) \leq \mathrm{UL}$

Step 2: Initialize the population.

Generate a random population according to the population size band number of design variables. For TLBO, the population size indicates the number of learners and the design variables indicate the subjects (i.e. courses) offered. This population is expressed as

population $=\left[\begin{array}{llll}x_{1,1} & x_{1,2} & \cdots & x_{1, D} \\ x_{2,1} & x_{2,2} & \cdots & x_{2, D} \\ \vdots & & \vdots & \vdots \\ x_{P_{n}, 1} & x_{P_{n}, 2} & \cdots & x_{P_{n}, D}\end{array}\right]$.

Step 3: Teacher phase.

Calculate the mean of the population column-wise, which will give the mean for the particular subject as $\mathrm{M}, \mathrm{D}=[\mathrm{m} 1, \mathrm{~m} 2, \ldots, \mathrm{mD}]$

The best solution will act as a teacher for that iteration Xteacher $=\mathrm{Xf}(\mathrm{X})=\min$

The teacher will try to shift the mean from $M, D$ towards X,teacher, which will act as a new mean for the iteration. So,

Mnew,D = Xteacher,D.

The difference between two means is expressed as Difference Mean(i) = r(i) [Mnew TF $\times$ M(i)]

Xnew,D = Xold,D + Difference,D

Accept Xnew if it gives better function value.

Step 4: Learner phase.

As explained above, learners increase their knowledge with the help of their mutual interaction

Step 5: Termination criterion.

Stop if the maximum generation number is achieved; otherwise repeat from Step 3. 
It is seen from the above steps that no provision is made to handle the constraints in the problem. Many types of constraint handling technique are available in the literature, such as incorporation of static penalties, dynamic penalties, adaptive penalties etc. Deb's heuristic constrained handling method is used in the proposed TLBO method. This method uses a tournament selection operator in which two solutions are selected and compared with each other.

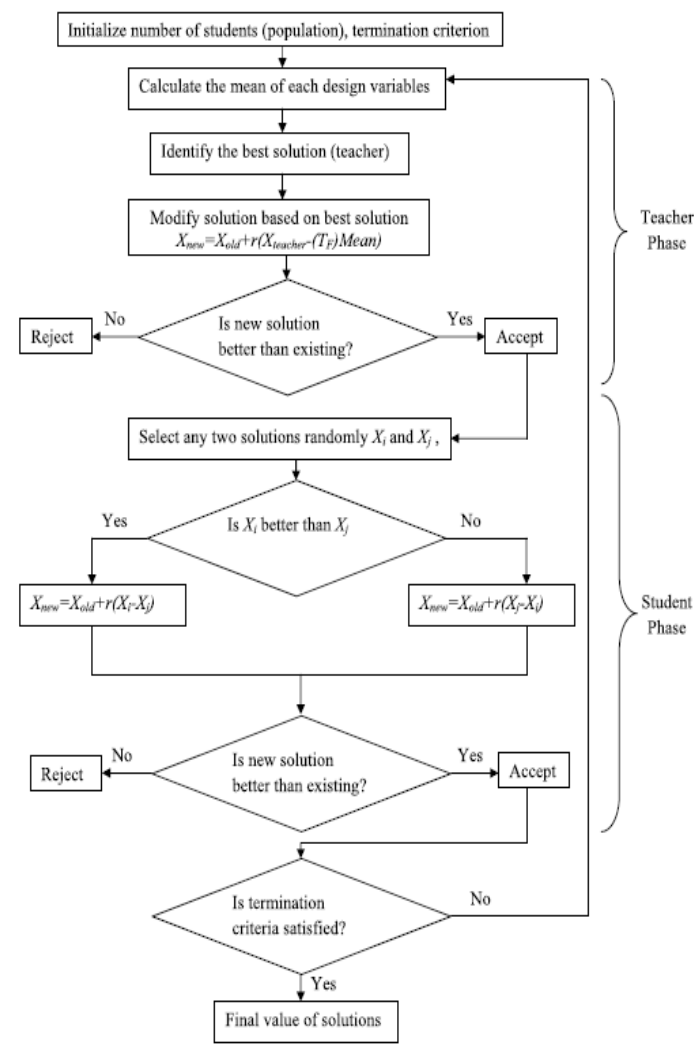

Fig.1 Flowchart of TLBO

The following three heuristic rules are implemented on them for the selection:

1. If one solution is feasible and the other infeasible, then the feasible solution is preferred.

2. If both the solutions are feasible, then the solution having the better objective function value is preferred. 3. If both the solutions are infeasible, then the solution having the least constraint violation is preferred.

To use TLBO, it is necessary to decide two variables $\mathrm{X}$ and $\mathrm{Y}$, where $\mathrm{X}$ is independent variable and $\mathrm{Y}$ is dependent variable of $X$. Therefore in order to find $a$ feasible solution using TLBO, first we have to define a function $\mathrm{F}(\mathrm{X}, \mathrm{Y})$ which will show the relation between $\mathrm{X}$ and Y.So from the data collected, we have considered,

$$
\mathbf{F}(\mathbf{X}, \mathbf{Y})=\frac{\mathbf{X}}{\mathbf{Y}}
$$

$\mathrm{X}=$ Number of activities performed on one station.
$\mathrm{Y}=$ Time required to do all activities at station.

Now, it is assumed that time required for each station is directly proportional to the number of activities of each station, we can define the relationship between $\mathrm{X}$ and $\mathrm{Y}$ as, Therefore, using this TLBO, we have to minimize the value of function $F(X, Y)$ in order to reduce cycle time. Doing TLBO iterations for teacher phase and student phase, two feasible solution will be obtained respectively. Comparing those two solution, the solution having minimum value of function is considered as optimal solution.

To do iterations using TLBO, though it is simple to calculate manually, a SCILAB code is generated. There are two phases involved in calculation, Teacher phase and Student phase. For student there are seven iterations, every station interaction with all other stations.

\subsection{Inputs required for iterations-}

To perform iterations few inputs are required like values of variable $\mathrm{X}$ and $\mathrm{Y}$, values of constants ' $\mathrm{r} 1$ ' and 'r2' , which are to be entered in console window of SCILAB. The fig. below shows the snapshoot of the console window where inputs are to given by user.

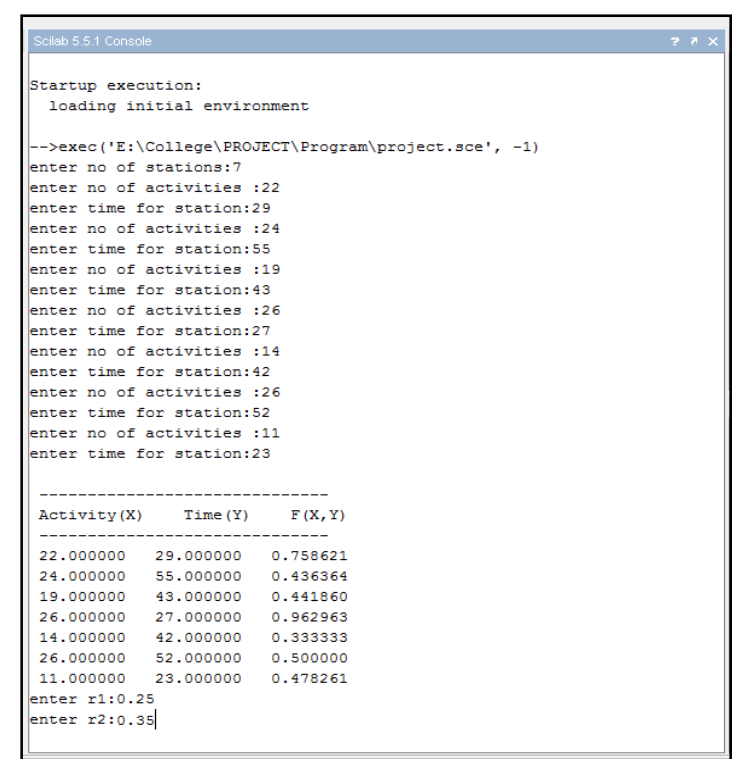

Fig.2 Console Window of SCILAB

\subsection{Output of SCILAB program}

Using TLBO formulae, the calculation for Teacher Phase and Student Phase is done by computer according to the inputs given by the user.Sample calculation for each phase is shown below:-

Teacher Phase:-

$$
\begin{aligned}
\text { Xnew } & =X+r 1(\text { Xbest }- \text { Xmean }) \\
& =22+0.25(14-19.85) \\
& =20.53
\end{aligned}
$$




$$
\begin{aligned}
\text { Ynew } & =Y+r 2(\text { Ybest }- \text { Ymean }) \\
& =29+0.35(42-380714) \\
& =30.1500
\end{aligned}
$$

\begin{tabular}{|c|c|c|}
\hline Activity (X) & Time (Y) & $\mathrm{F}(\mathrm{X}, \mathrm{Y})$ \\
\hline 20.535714 & 30.150000 & 0.681118 \\
\hline 22.535714 & 56.150000 & 0.401348 \\
\hline 17.535714 & 44.150000 & 0.397185 \\
\hline 24.535714 & 28.150000 & 0.871606 \\
\hline 12.535714 & 43.150000 & 0.290515 \\
\hline 21.535714 & 53.150000 & 0.405187 \\
\hline 9.535714 & 24.150000 & 0.394854 \\
\hline \multicolumn{3}{|c|}{$F \min =0.290515$} \\
\hline
\end{tabular}

\section{Fig.3 Teacher Phase}

Similarly for Student Phase, sample calculation is done below:-

\section{For 1st iteration}

\begin{tabular}{|c|c|c|}
\hline Activity (X) & Time (Y) & $F(X, Y)$ \\
\hline 20.535714 & 30.150000 & 0.681118 \\
\hline 23.035714 & 65.250000 & 0.353038 \\
\hline 18.285714 & 49.050000 & 0.372797 \\
\hline 25.535714 & 28.850000 & 0.885120 \\
\hline 14.535714 & 47.700000 & 0.304732 \\
\hline 21.785714 & 61.200000 & 0.355976 \\
\hline 12.285714 & 26.250000 & 0.468027 \\
\hline
\end{tabular}

$$
\begin{aligned}
X 2 \text { new } & =X+r 1|X 1-X 2| \\
& =22.53+0.25|20.53-22.53| \\
& =23.03
\end{aligned}
$$

$$
\begin{aligned}
Y 2 \text { new } & =Y+r 2 / Y 1-Y 2 \mid \\
& =56.15+0.35|30.150-56.15| \\
& =65.25
\end{aligned}
$$

\section{Fig.4 Student Phase}

In this way, for every station the iteration are carried. So in all for seven stations, perform seven iterations of student phase. From above iterations, the conclusions are that the minimum value of function was obtained at station no. 5 for all iterations. Therefore, for station no. 5 cycle time is $42 \mathrm{sec}$. This is considered as optimal cycle time for each station.

\section{Results}

Objective function was to minimize cycle time and number of workstations in order to ensure a smooth distribution of workload between workstations. Teaching-learning based optimization algorithm is used to obtain high quality solutions, in a very short computational time. In this case, the optimal solution for the cycle time with lower smoothness index is obtained. The optimal cycle time came out to be $42 \mathrm{sec}$. Therefore it is required to balance all other station in such a way that the time required for each station should be near to 42 seconds.

\section{Conclusion}

A real life Assembly line balancing problem with additional assignment restrictions is a focus of this study. Thus, number of tasks in addition to precedence and cycle time constraints are taken into account, when the allocation of the tasks to an ordered sequence of workstations is determined. Moreover, the problem considered, involves several compatible and incompatible zoning constraints. Accordingly, some groups of tasks must be executed together on the same station (compatible tasks) and other tasks were prevented from being assigned to the same station (incompatible tasks). Finally, the problem is also composed of several tasks which should be assigned to the different stations with no other tasks assigned (negative zoning constraint).

Moreover, teaching-learning based optimization algorithm is a quite new method within the other meta-heuristics methods. The literature review shows that this algorithm gives improved results. As a further research area, its phases can be improved using different teachers in teacher phase and using tutorials or self-learning capability in learner phase.

\section{References}

Hindriyanto Dwi Purnomo, Hui-Ming Wee (2014). Maximizing production rate and workload balancing in a two-sided assembly line using Harmony Search Computers \& Industrial Engineering 76, 222-230.

Simaria Ana S. , Vilarinho Pedro M. (2009), 2-ANTBAL: An ant colony Optimization Algorithm for balancing two-sided assembly lines Computers \& Industrial Engineering 56,489-506.

Rao R.V, Savsani V.J. and Vakharia D.P. (2011), Teachinglearning-based optimization: A novel method for constrained mechanical design optimization problems, Elsevier, 303-315.

Parames Chutima Wanwisa Naruemitwong (2014), A Pareto biogeography-based optimisation for multi-objective twosided assembly line sequencing problems with a learning effect Computers \& Industrial Engineering 89-104.

Simaria Ana S. ,Pedro M. Vilarinho (2009), 2 ANTBAL: An ant colony optimisation algorithm for balancing two-sided assembly lines Computers \& Industrial Engineering 56, 489-506

Adil Baykasog lu, Alper Hamzadayi, Simge Yelkenci Kose(2014), Testing the performance of teaching-learning based optimization (TLBO) algorithm on combinatorial problems:Flow shop and job shop scheduling cases Information Sciences 276-218.

Rao R. Venkata, Kalyankar V.D., Waghmare G. (2014), Parameters optimization of selected castin processes using teaching-learning-based optimization algorithm Applied Mathematical Modelling 38-5608.

Rao R. Venkata n, Kalyankar V.D. (2013), Parameter optimization of modern machining processes using teaching-learning-based optimization algorithm Engineering Applications of Artificial Intelligence 26-531.

Rao R. Venkata and Kalyankar V.D. Multi-pass turning process parameter optimization using teaching-learningbased optimization algorithm Scientia Iranica 20 (3), 967 974. 